\title{
Simultaneous Breast Cancer and Kaposi's Sarcoma Complicating Rheumatoid Arthritis
}

\author{
Frida Barak $^{\mathrm{a}}$ Tatiana Reitblat ${ }^{\mathrm{b}}$ \\ ${ }^{\mathrm{a}}$ Oncology Institute, and ${ }^{\mathrm{b}}$ Rheumatology Outpatient Clinic, Barzilai Medical \\ Center, Ashkelon, Affiliated to the Faculty of Health Sciences, Ben-Gurion \\ University of the Negev, Beersheba, Israel
}

\section{Key Words}

Secondary cancers · Rheumatoid arthritis - Methotrexate · Vinorelbine · Kaposi's sarcoma

\begin{abstract}
For a number of years we have been following the medical literature to find a relationship between chronic treatment with methotrexate and breast cancer occurrence, because we had had in our clinic a female patient who had had two consecutive cancers following methotrexate treatment for rheumatoid arthritis (RA). We were much surprised to find in some papers that breast cancer incidence is low in women with RA. Since then, we have found several papers explaining the low incidence of breast cancer among women being under NSAIDs, but those papers are not univocal. Methotrexate is a known antifolate agent and it has been demonstrated that dietary shortage of folate is a risk factor for breast cancer development.
\end{abstract}

\section{Introduction}

In the last two decades there has been a growing interest in studying the appearance of secondary malignancies in conjunction with rheumatoid arthritis (RA) [1, 2]. The majority of the described malignancies included hematological malignancies especially lymphoma, lung cancer, melanoma and Kaposi's sarcoma [1-5]. Breast cancer incidence is not considered to be increased in RA, except, possibly, in Japanese women [3]. 


\section{Case Report}

Our interest in the subject of secondary tumors in patients being treated chronically with immunosuppressive therapy, especially with methotrexate, started with the presentation of a 78year-old woman in our Oncology Institute. This patient had been treated for RA with small doses of prednisolone, up to $10 \mathrm{mg}$ daily, and methotrexate, up to $12.5 \mathrm{mg}$ once a week, for a period of 20 years. She was then diagnosed with stage II infiltrating ductal carcinoma of the breast (T2N0M0), containing high levels of estrogen and progesterone receptors. Her general condition at that time was considered good and her Karnovsky status was $90 \%$. Postoperative adjuvant treatment was commenced with tamoxifen and the therapy with prednisolone and methotrexate was continued unchanged. For 2 years after her breast cancer diagnosis, this patient continued to be without any evidence of breast cancer recurrence and she was free of any RA activity as well. At that time, Kaposi's sarcoma lesions of the right inner thigh skin and foot were identified and confirmed by biopsy. It was then decided to cease the immunosuppressive therapy and to give the patient the opportunity to recover from her Kaposi's sarcoma. Due to termination of prednisolone and methotrexate and in order to change previous immunosuppressive treatment, tetracycline (minocycline) and gold salts (Ridaura) were begun. As a result, almost a month later, a spontaneous resolution of some of the Kaposi's lesions was observed. Some of the lesions shrunk and some even disappeared. This response was observed for a period of 30 days but as soon as the lesions started to regrow, a weekly treatment with vinblastine was started. Soon after the initiation of chemotherapy, our patient on her own volition stopped tamoxifen that had been prescribed to her earlier.

After a while, lung metastases, confirmed by histology to be of breast cancer origin, were identified. Consequently, she was recommended to initiate an additional hormonal agent, idoxifene. Unfortunately, she died of lung insufficiency due to breast cancer metastases to her lungs. She did not have any signs of Kaposi's sarcoma at the time of her death.

Notably, with the vinblastine treatment a complete response was obtained in Kaposi's sarcoma, lasting for 1 year.

\section{Discussion}

We reported this case since we had to optimize several clinical judgments regarding a combined approach to a multiple simultaneous problem, using 1 drug applicable for 2 malignancies, breast carcinoma and Kaposi's sarcoma. Our experience illustrated that even in the period of well-established guidelines, when clinicians are facing a special situation, they may sometimes have to make an individual clinical decision that matches a specific patient. In general, Vinca rosea derivates, especially vincristine and vinorelbine, are the chemotherapy protocols recommended for recurrent Kaposi's sarcoma. Vinblastine is usually not used. Our fear that our patient, affected by chronic methotrexate use, might develop neutropenic fever, led to the clinical decision of using vinblastine rather than the other derivates, since vinblastine is in use for breast cancer treatment. Lately, neither signs of neutropenic fever, nor of Kaposi's sarcoma were confirmed.

Our wish to discuss this issue is generated by the controversial data. On the one hand, methotrexate is a recognized antifolate agent and chronic lack of folates is an accepted factor in breast cancer pathogenesis [6]. Yet, on the other hand, antiinflammatory non-steroidal drugs may reduce the risk of carcinogenesis in general, and of breast cancer specifically, during the lifetime [1-4]. Methotrexate has been used for many years in RA and in recent years a supplement of folinic acid is recommended by rheumatologists. In April 2005, the British Journal of Cancer published the first study which explored the level of DNA damage and folate status in mononuclear cells in 
breast cancer and benign breast disease patients, providing some evidence that reduced folate may be implicated in the development of breast cancer [7].

Based on retrospective studies published in different journals it is indicated clearly that RA patients have a lower incidence of breast cancer, probably due to the fact that they are being treated with NSAIDs [6], even though we discovered that this issue is not univocal. In January 2009, an additional paper entitled 'Use of Aspirin, Other Nonsteroidal Anti-Inflammatory Drugs, and Acetaminophen and Risk of Breast Cancer among Premenopausal Women in the Nurses' Health Study II' by Eliassen et al. was published in Archives of Internal Medicine. This paper supported the contrary claim that 'chemopreventive use of aspirin or other NSAIDs for breast cancer among premenopausal women is not warranted' [8].

\section{Conclusion}

Further prospective investigation is needed to explain and prove the association between immune diseases, immunosuppressive treatment with methotrexate, breast cancer, and Kaposi's sarcoma. However, despite the lack of knowledge at present, one should keep in mind the possible link among these conditions, before prescribing prolonged immunosuppressive treatment.

\section{References}

-1 Cibere J, Sibley J, Haga M: Rheumatoid arthritis and the risk of malignancy. Arthritis Rheum 1997;40:1580-1586.

-2 Chakravarty EF, Genovese MC: Associations between rheumatoid arthritis and malignancy. Rheum Dis Clin North Am 2004;30:271-284.

-3 Smitten AL, Simon TA, Hochberg MC, Suissa S: A meta-analysis of the incidence of malignancy in adult patients with rheumatoid arthritis. Arthritis Res Ther 2008;10:R45.

4 Bernatsky S, Clarke AE, Suissa S: Hematologic malignant neoplasms after drug exposure in rheumatoid arthritis. Arch Intern Med 2008;168:378-381.

5 Buchbinder R, Barber M, Heuzenroeder L, et al: Incidence of melanoma and other malignancies among rheumatoid arthritis patients treated with methotrexate. Arthritis Rheum 2008;59:794-799.

6 Lajous M, Romieu I, Sabia S, Boutron-Ruault MC, Clavel-Chapelon F: Folate, vitamin B12 and postmenopausal breast cancer in a prospective study of French women. Cancer Causes Control 2006;17:1209-1213.

-7 Hussien MM, McNulty H, Armstrong N, Johnston PG, Spence RA, Barnett Y: Investigation of systemic folate status, impact of alcohol intake and levels of DNA damage in mononuclear cells of breast cancer patients. Br J Cancer 2005;92:1524-1530.

8 Eliassen AH, Chen WY, Spiegelman D, Willett WC, Hunter DJ, Hankinson SE: Use of aspirin, other nonsteroidal anti-inflammatory drugs, and acetaminophen and risk of breast cancer among premenopausal women in the Nurses' Health Study II. Arch Intern Med 2009;169:115-121; discussion 121 